\title{
A single-centre hospital-wide handoff standardisation report: what is so special about that?
}

\section{Maitreya Coffey, ${ }^{1,2,3}$ Lennox Huang $^{1}$}

\begin{abstract}
'Department of Paediatrics, Hospital for Sick Children University of Toronto, Toronto, Ontario, Canada

${ }^{2}$ Centre for Quality Improvement and Patient Safety, University of Toronto, Toronto, Ontario, Canada

${ }^{3}$ Children's Hospitals Solutions for Patient Safety http://www. solutionsforpatientsafety.org
\end{abstract}

\section{Correspondence to} Dr Maitrey Coffey, Centre for Quality Improvement and Patient Safety, 525 University Avenue, Suite 630, Toronto, Ontario, Canada M5G 1X8; trey.coffey@sickkids.ca

Accepted 4 April 2017 Published Online First 21 April 2017

\section{SLinked}

http://dx.doi.org/10.1136/ bmjqs-2016-006195

\section{CrossMark}

To cite: Coffey M, Huang L. BMJ Qual Saf

2017;26:698-700.
Healthcare leaders and scholars have articulated gaps in handoff quality across nearly all healthcare settings. A variety of drivers, including hospital accreditation, internal and external safety event analyses and medical education objectives, have given rise to a proliferation of imperatives to improve this situation. Healthcare leaders have developed a greater appreciation that handoff is a key component of a larger set of culture and teamwork strategies that are necessary to reduce harm. Researchers and medical educators have created handoff programmes, provided empirical evidence for their positive impact on safety and worked tirelessly to disseminate them. ${ }^{1}{ }^{2}$ Quality improvers from a variety of disciplines have begun to adapt and apply standardised handoff in an increasingly diverse array of settings.

In light of this, one might think it less than noteworthy to discover a report of a single institution's hospital-wide handoff standardisation programme. ${ }^{3}$ To the contrary, we find this report by Shahian et al ${ }^{3}$ novel and rich with important messages. We agree with their assertion that this is the largest single-institution implementation of the I-PASS handoff system ${ }^{2}$ reported in a tertiary general hospital, in this case, Massachusetts General Hospital, which has 25000 employees. Using a relatively low-cost approach, they managed to implement the system across 15 medical departments, as well as nursing, train nearly 6000 healthcare staff and collect observational data on process reliability at baseline and over 7 months of implementation.

Our combined experience in multiple organisations has afforded us opportunities to understand and engage with the effort to improve handoff from multiple vantage points, including through participation as a site in the I-PASS study. ${ }^{2}$ Through clinical, research and academic and hospital leadership roles, we have seen successes and challenges locally, regionally and internationally.

So, why are we so interested in this paper? The reduction in preventable adverse events reported in the I-PASS study $^{2}$ has generated welcome traction for improving handoff in general and for the I-PASS approach in particular. Many individuals and organisations find themselves wishing, planning or attempting to implement standardised handoff organisation-wide, but find it challenging, if not Herculean, to do so. It is rare to encounter a hospital which has successfully implemented and evaluated standardised handoff across the full scope of disciplines, subspecialties and workflows. Shahian $e t a l^{3}$ have provided an account of how they anticipated and endeavoured to address many of the factors that make it difficult to achieve organisation-wide standardised handoff. We feel they have key messages with broad relevance to the patient safety and quality improvement communities.

First, there is the matter of organisational leadership selecting this intervention among the dizzying array of potential quality and safety improvement targets competing for (severely) limited attention and resources. The authors have synthesised multiple information sources, including culture surveys (we all do them ... how often do we take specific action based on the data?), and both hard and soft intelligence ${ }^{4}$ on the magnitude of handoff quality problems. This appeals, because it suggests the possibility that the seemingly infinite number of safety problems may converge around a smaller number of fundamental processes or behaviours with the potential to mitigate or exacerbate risk across all situations.

In many ways, the paper by Shahian et al reads as a classic change 
management text. Gaining support from the top, creating a burning platform, applying a multimodal strategy, finding local champions and linking their work to other change initiatives are all effective strategies that can be found in many popular change management books. ${ }^{5}$ We know that applying some combination of these strategies can result in successful change in the behaviour of healthcare professionals. ${ }^{6}$ However, examples of successful applications of these techniques in healthcare, especially in the setting of a large complex teaching hospital, remain uncommon. Large healthcare organisations are often decentralised by necessity with individual units and programmes and with processes specific to their patient population. Achieving alignment and buy-in around initiatives can be even more difficult in decentralised structures and often requires a parallel organisational structure to achieve change.

The leaders of this initiative must have navigated considerable obstacles despite what appears to be a highly effective organisational structure for decisionmaking and oversight. Despite their steadfast commitment to using evidence and adhering to fundamental safety principles, we can be sure that they encountered individuals and groups who felt the standardised tool-despite the leaders' assertion that it was 'simple, intuitive and compatible'-was not for them and did not meet their unique and special needs, or that it was unnecessary given their 'unwarranted comfort with current approaches'. A fine balance between topdown pressure and stakeholder engagement and choice appears to have been particularly well struck. This balancing act continued as the effort unfolded, as they found compromise between standardisation (in particular maintaining fidelity to an intervention as carried out in the research cited) and end-user demand to adapt the programme to local workflows and to receive education that was multimodal and flexible in duration and format.

Leadership support is nearly always cited as a success factor in QI accounts, but this case contains some specifics worth noting. The CEO personally delivered the message executives were highly visible in the marketing and communication strategy; and executives themselves participated in all of the teaching, which ultimately reached nearly 6000 staff. Senior leadership going beyond endorsement to direct participation may distinguish their approach from others who are trying to accomplish what these authors have.

The authors mention the challenge of applying and scaling up interventions studied in a rigorous research setting to the real world. Evaluating a programme without the benefit of research funding is only one aspect of this challenge. In the example described in this paper, they found a highly pragmatic strategynamely a distributed network of unit-level champions using simple tools-to gather considerable amount of process data using the sound quality improvement strategy of multiple small samples over time. Anyone who regards these steps as routine or easy to achieve has probably not personally tried to convince people to make time for observations of handoffs, which occur at either end of a very long healthcare workday. It is quite impressive that all but one unit submitted some process observation data. With this hands-on, manual strategy, data collection likely played a role beyond simply capturing observations. Data collected also provided an opportunity to demonstrate importance and to occasion meaningful conversations between champions and a great number of front-line providers.

Some may assert that the efforts reported by Shahian $e t a l^{3}$ did not achieve high levels of adherence, but this did appear to improve markedly from baseline and was sustained over a period of time. It is also important to note that the level of adherence demonstrated here is similar if not greater than that achieved in the original I-PASS study, ${ }^{2}$ making it plausible that they may also realise the associated safety improvements seen in that study. The authors indicate the depth of organisational commitment, signal a sophisticated understanding of the effort required to achieve lasting attitude and behaviour change, and send a key message to others by describing this intensive 3-year programme as 'phase one' and acknowledging that long-term sustainability remains an open question, are planning for a further 3-year 'phase two' for additional spread and sustainability work. ${ }^{78}$

What does all of this tell us about our prospects for achieving widespread adoption of standardised handoff? Shahian $e t$ al have given us a blueprint for how to achieve change in a complex environment. The obstacles and challenges they encountered will not be going away anytime soon. We have much more to learn about how professionals who have been trained in a culture emphasising autonomy, authority and individualism can embrace the standardised communication methods universally accepted in the industries we attempt to learn from and emulate. In the meantime, it seems good oldfashioned leadership will and application of tried and true change management and quality improvement strategies can go a long way towards getting us started.

Competing interests None declared.

Provenance and peer review Commissioned; internally peer reviewed.

\section{REFERENCES}

1 Arora VM, Manjarrez E, Dressler DD, et al. Hospitalist handoffs: a systematic review and task force recommendations. J Hosp Med 2009;4:433-40.

2 Starmer AJ, Spector ND, Srivastava R, et al. Changes in medical errors after implementation of a handoff program. N Engl J Med 2014;371:1803-12.

3 Shahian DM, McEachern K, Rossi L, et al. Large-scale implementation of the I-PASS handover system at an academic medical center. BMJ Qual Saf 2017;26:760-70. 


\section{Editorial}

4 Martin GP, McKee L, Dixon-Woods M. Beyond metrics? Utilizing 'soft intelligence' for healthcare quality and safety. Soc Sci Med 2015;142:19-26.

5 Kotter JP. Leading change. Harvard Business School Press, 1996. ISBN 978-0-87584-747-4.

6 Colquhoun HL, Squires JE, Kolehmainen N, et al. Methods for designing interventions to change healthcare professionals' behaviour: a systematic review. Implement Sci 2017;12:30.

7 Greenhalgh T, Robert G, Macfarlene F, et al. Diffusion of innovations in service organizations: Systematic review and recommendations. Millbank Q 2004;82:581-629.

8 May CR, Mair F, Finch T, et al. Development of a theory of implementation and integration: Normalization Process Theory. Implement Sci 2009;4:1-9. http://dx.doi.org/ 10.1186/1748-5908-4-29. 